\title{
VARIABILIDADE ESPACIAL DOS ATRIBUTOS DO SOLO SOB AGROFLORESTA NA REGião de Humaitá, AM
}

\section{Marcelo Dayron Rodrigues Soares ${ }^{1 *}$, Milton César Costa Campos ${ }^{1}$, Ivanildo Amorim de Oliveira ${ }^{2}$, José Maurício da Cunha ${ }^{1}$, Zigomar Menezes de Souza ${ }^{3}$, Renato Eleotério Aquino ${ }^{4}$, Douglas Pinheiro da Silva ${ }^{1}$, Julimar Fonseca Silva ${ }^{1}$}

\author{
${ }^{1}$ Universidade Federal do Amazonas, UFAM, Humaitá, AM-Brasil, CEP: 69.800-000. \\ ${ }^{2}$ Instituto Federal de Educação, Ciência e Tecnologia do Pará, IFPA, Breves, PA-Brasil, CEP: 68.800-000 \\ ${ }^{3}$ Universidade Estadual de Campinas, UNICAMP, Campinas, SP-Brasil, CEP: 13083-875 \\ ${ }^{4}$ Universidade Estadual Paulista, UNESP, Jaboticabal, SP- Brasil, CEP: 14.884-900 \\ * Autor para correspondência: marcelo.dayron@gmail.com
}

Recebido em 22 de maio de 2017. Aceito em 25 de fevereiro de 2018. Publicado em 06 de abril de 2018.

\begin{abstract}
REsumo - Os sistemas agroflorestais visam a constituir um ambiente natural com aumento da diversidade e, desta maneira, estes sistemas podem ser empregados como ecossistemas sustentáveis em ambientes modificados. O objetivo deste trabalho foi avaliar a variabilidade espacial dos atributos do solo de um Argissolo Vermelho Alítico plíntico sob agrofloresta na região de Humaitá, AM. Foram georreferenciados 64 pontos, em um grid amostral com as dimensões 70 × $70 \mathrm{~m}$, e o intervalo entre os pontos de $10 \mathrm{~m}$. Nos cruzamentos entre os pontos, foram coletadas amostras de solos deformadas e indeformadas na profundidade 0-0,20 m. Em cada amostra, foi analisado a densidade do solo (DS), macroporosidade (Macro), microporosidade (Micro), porosidade total (PT), diâmetro médio geométrico (DMG), pH, matéria orgânica (MO), P, K, Mg, H + Al. A variabilidade espacial desses atributos foi analisada por meio de medidas descritivas e técnicas geoestatísticas. Todos os atributos estudados apresentaram dependência espacial. Os resultados apresentaram baixa variação dos atributos, forte a moderada o grau de dependência e os valores de alcance maior que estipulado no espaçamento. O modelo esférico do semivariograma escalonado representou melhor a distribuição espacial dos atributos.
\end{abstract}

Palavras-chave: Dependêncla Espaclal; Geoestatística; Uso do Solo.

\section{SPATIAL VARIABILITY OF SOIL ATTRIBUTES UNDER AGROFORESTRY IN THE HUMAITÁ REgION, AM}

Aвstract - The agroforestry systems aim to constitute a natural environment with increased diversity and, in this way, these systems can be used as sustainable ecosystems in modified environments. The objective of this study was to evaluate the spatial variability of soil attributes of a plinthic Red Alizol under agroforestry in the region of Humaitá, AM. In a sample grid with the dimensions of $70 \times 70 \mathrm{~m}$ and the interval of $10 \mathrm{~m}$ between the points, 64 points were georeferenced. At the intersections between the points, samples of deformed and undeformed soils were collected at depth of $0-0.20 \mathrm{~m}$. Soil density (SD), macroporosity (Macro), microporosity (Micro), total porosity (TP), geometric mean diameter (GMD), $\mathrm{pH}$, organic matter $(\mathrm{OM}), \mathrm{P}, \mathrm{K}, \mathrm{Mg}, \mathrm{H}+\mathrm{Al}$ were analyzed in each sample. The spatial variability of these attributes was analyzed by descriptive measures and geostatistical techniques. All analyzed attributes presented spatial dependence. The results showed low variation of the attributes, strong to moderate degree of dependence and values of greater reach that stipulated in the spacing. The spherical model of the scaled semivariogram represented better spatial distribution of the attributes.

Keywords: Spatial Dependence; Geostatistics; Soil Use. 


\section{VARIAbilidad ESPacial de los ATributos del SuElo bajo Sistema Agroforestal en la Región de Humaitá, AM}

RESUMEN - Los sistemas agroforestales apuntan a crear un ambiente natural con aumento de la diversidad y, de esta manera, estos sistemas pueden ser empleados como ecosistemas sostenibles en ambientes modificados. El objetivo de este trabajo fue evaluar la variabilidad espacial de los atributos del suelo de un Acrisol Rojo Alítico plíntico bajo sistema agroforestal en la región de Humaitá, AM. Se georreferenciaron 64 puntos, en una cuadrícula de muestreo con las dimensiones 70 x 70 $\mathrm{m}$, y el intervalo entre los puntos de $10 \mathrm{~m}$. En los cruces entre los puntos, se recolectaron muestras de suelos deformadas e inalteradas en la profundidad 0-0,20 m. En cada muestra, se analizó la densidad del suelo (Ds), la macroporosidad (Macro), la microporosidad (Micro), porosidad total (PT), diámetro medio geométrico (DMG), pH, materia orgánica (MO), P, K, Mg , H + Al. La variabilidad espacial de estos atributos fue analizada por medio de medidas descriptivas y técnicas geoestadísticas. Todos los atributos estudiados presentaron dependencia espacial. Los resultados presentaron baja variación de los atributos, fuerte a moderado el grado de dependencia y los valores de alcance mayor que estipulado en el espaciamiento. El modelo esférico del semivariograma escalonado representó mejor la distribución espacial de los atributos.

Palabras clave: Dependencla Espaclal; Geoestadística; Uso del Suelo.

\section{INTRODUÇÃO}

A região Amazônica, em sua maior parte, é caracterizada por solos ácidos, com baixa fertilidade e baixa capacidade de troca catiônica (Cunha et al. 2007). A conversão de florestas amazônicas para atividades florestais e agropecuárias, resulta em mudanças nos atributos do solo que, em muitos casos, conduzem a perda da produtividade. Esse fato, é atribuído, a destruição d estrutura do solo (Chauvel et al. 1991). Marques et al. (2004), em estudos com atributos físicos, químicos e hídricos, em sistema agroflorestal na região Amazônica, obtiveram bons resultados para os atributos estudados, destacando que, a retenção hídrica demonstra que o solo tem a capacidade de reter elevado teor de umidade.

Os sistemas multiestratificados na Amazônia são cultivadas por agricultores familiares com fins comercias e para subsistência (Castro et al. 2009). Da mesma maneira, Viera et al. (2007) afirmam que é comum a prática de cultivar espécies frutíferas em sistemas agroflorestais estes realizados por agricultores familiares com fins especialmente de sua subsistência.

Por outro lado, a variabilidade espacial dos atributos dos solos é resultado de processos pedogenéticos e pode ser demonstrada por resultados dos levantamentos e análises dos solos, bem como pelas diferenças encontradas nas produções das plantas (Silva et al. 2010). Vale ressaltar que a formação do solo é resultante de uma série de fatores e o produto é bastante heterogêneo em relação as suas características e propriedades (Cajazeiras e Assis Júnior 2011). Já Bottega et al. (2013) afirmam que é indispensável a caracterização da variabilidade espacial dos atributos químicos e físicos do solo por meio de amostragem capaz de representar tais variações.

Diversos trabalhos com variação espacial em diferentes ambientes no Sul do Amazonas, foram realizados e, mostraram resultados satisfatórios, os quais, podemos cita: floresta (Campos et al. 2013); cana-de-açúcar (Oliveira et al. 2014); terra preta de índio sob pastagem (Soares et al. 2015); terra preta de índio sob cultivo de cacau (Silva et al. 2016), e entre outros. Com base nessas informações, e com o auxílio da geoestatística, a estrutura de dependência espacial dos atributos do solo vem sendo intensamente estudada e modelada, permitindo a sua visualização espacial.

A presente pesquisa teve como objetivo avaliar a variabilidade espacial dos atributos do solo de um Argissolo 
Vermelho sob agrofloresta em Humaitá, AM.

\section{MATERIAL E MÉTODOS}

A área de estudo está localizada no município de Humaitá, sul do Estado do Amazonas, situada sob as coordenadas geográficas de “7³0’24” de latitude sul e 6304”56" longitude oeste, altitude média de 63 m. O clima da região, segundo a classificação de Köppen, é do tipo tropical chuvoso, com período seco de pequena duração (Am), temperaturas oscilando entre 25 e $27^{\circ} \mathrm{C}$ e precipitação média anual de $2.500 \mathrm{~mm}$, com período chuvoso iniciando em outubro e estendendo-se até junho e com a umidade relativa do ar variando entre 85 e 90\% (Brasil, 1978). O solo da área foi classificado como Argissolo Vermelho Alítico plíntico.

A textura do solo foi realizada pelo método da pipeta, conforme a metodologia da Embrapa (2011), sendo os seguintes resultados: 220, $79 \mathrm{~g} \mathrm{Kg}^{-1}$; 230,11 $\mathrm{g} \mathrm{Kg}^{-1}$; $549 \mathrm{~g} \mathrm{Kg}^{-1}$, respectivamente para areia, silte e argila.

Essa área vem cerca de 20 anos, sendo cultivada com café (Coffea sp), cacau (Theobroma cacao), palmeiras (Arecaceae), andiroba (Carapa guianensis) entre outras. O sistema agroflorestal adotado na área de estudo, é multiestratificados, uma vez que os agricultores familiares, que implantaram tais espécies, visam sobretudo, a colheita de frutos para a venda. Vale ressaltar, que segundo Vieira et al. (2007), os sistemas agroflorestais na região, são manejados, em sua maioria, de forma tradicional e com baixo nível tenológico.

Foi demarcada uma área de $4900 \mathrm{~m}^{2}(70$ x 70 m) que continham 64 pontos georreferenciados dispostos em malha de $10 \times 10 \mathrm{~m}$. Em cada ponto, o solo foi amostrado na profundidade $0,20 \mathrm{~m}$, conforme localização da área de estudo Figura 01.

Figura 1 - Mapa de localização da área de estudo no município de Humaitá-AM.

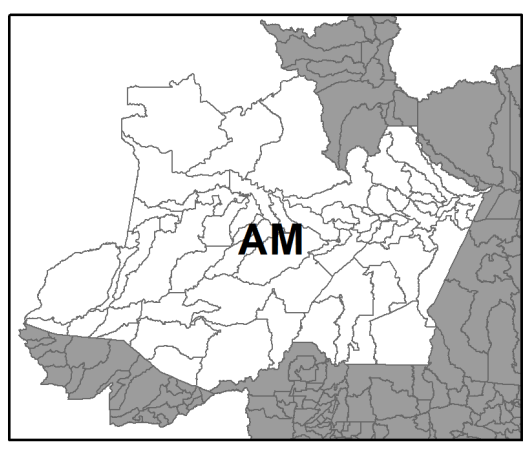

Legenda
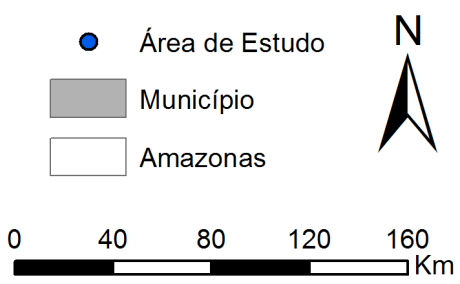

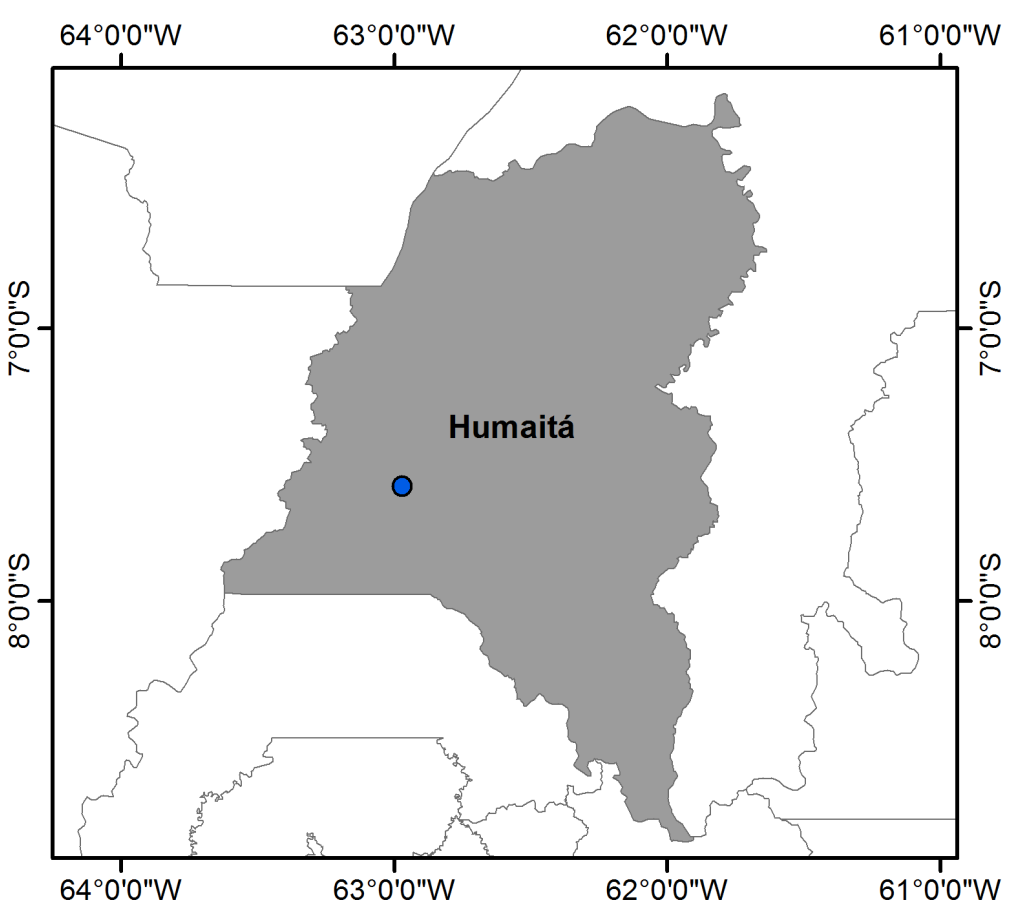

Foram abertas trincheiras de $0,30 \mathrm{~m}$ de profundidade $(0,3 \times 0,3 \mathrm{~m}$ de largura) em cada ponto da malha estudo, para coleta das amostras de formadas e indeformadas em anéis volumétricos de 0,05 $\mathrm{m}$ de diâmetro e $0,05 \mathrm{~m}$ de altura. A microporosidade foi determinada em mesa de tensão e correspondeu à umidade volumétrica da amostra submetida a uma tensão de $0,006 \mathrm{MPa}$, após saturação. A porosidade total e a densidade do solo 
foram obtidas segundo Embrapa (1997), e a macroporosidade foi obtida por diferença entre a porosidade total e a microporosidade. A densidade do solo (Ds) foi calculada pela relação entre a massa seca a $105{ }^{\circ} \mathrm{C}$ durante 24 h da amostra de solo do cilindro volumétrico (Embrapa, 2011).

Nos pontos de cruzamento da malha, foram coletadas amostras deformadas na profundidade $0,0-0,20 \mathrm{~m}$ para determinação do diâmetro médio geométrico (DMG). As amostras foram secadas a sombra, levemente destorroadas, e passadas em peneira de 9,51 $\mathrm{mm}$ e retidas em peneiras de 4,72 $\mathrm{mm}$ de diâmetro de malha, para as análises relativas à agregação. A separação e estabilidade dos agregados foram determinadas segundo Kemper e Chepil (1965). Os agregados inicialmente foram umedecidos e posteriormente colocados em contato com a água sobre a peneira de 2,00 $\mathrm{mm}$ por quinze minutos, a massa do material retido em cada peneira, e colocada em estufa a $105^{\circ} \mathrm{C}$.

A análise laboratorial dos atributos químicos foi realizada de acordo com o manual proposto pela Embrapa (1997) sendo analisados os seguintes atributos: magnésio $(\mathrm{Mg})$, fósforo $(\mathrm{P})$, potássio $(\mathrm{K}), \mathrm{pH}$, matéria orgânica (MO), acidez potencial $\left(\mathrm{H}+\mathrm{Al}^{3+}\right)$.

A estatística descritiva foi usada para resumir os dados, sendo calculado a média, mediana, desvio padrão, coeficiente de variação, coeficiente de assimetria e de curtose. A hipótese de normalidade dos dados foi testada pelo teste de Kolmogorov-Smirnov, no software estatístico Minitab 14.

Para a caracterização da variabilidade espacial, foi utilizada a análise geoestatística (Isaak e Srivastava, 1989). Sob teoria da hipótese intrínseca o semivariograma experimental foi estimado pelo método clássico por meio do estimador (Equação 1):

$$
\hat{Y}(h)=\frac{1}{2 N(h)} \sum_{i=1}^{N(h)}\left[Z\left(x_{i}\right)-Z\left(x_{i}+h\right)\right]^{2}
$$

Sendo: $\gamma(h)$ - valor da semivariância para uma distância $h ; N(h)$ - número de pares envolvidos no cálculo da semivariância; $\mathrm{Z}(\mathrm{xi})$ - valor do atributo $\mathrm{Z}$ na posição $\mathrm{x}_{\mathrm{i}} ; \mathrm{Z}\left(\mathrm{x}_{\mathrm{i}}+\mathrm{h}\right)$ - valor do atributo $\mathrm{Z}$ separado por uma distância $h$ da posição $x_{i}$.

$\mathrm{Na}$ análise do grau de dependência espacial das variáveis em estudo, utilizou-se a classificação de Cambardella et al. (1994), onde valores de $\left[\left(\mathrm{C}_{0} /\left(\mathrm{C}_{0}+\mathrm{C}_{1}\right)\right]<25 \%\right.$ são considerados dependência espacial forte, valores de $\left[\left(\mathrm{C}_{0} /\right.\right.$ $\left.\left(\mathrm{C}_{0}+\mathrm{C}_{1}\right)\right]$ entre 25 e $75 \%$ indicam dependência espacial moderada e $\left[\left(\mathrm{C}_{0} /\left(\mathrm{C}_{0}+\mathrm{C}_{1}\right)\right]>\right.$ que $75 \%$ dependência espacial fraca.

O ajuste do modelo matemático aos semivariogramas experimentais foi por meio do software Gs+ (Robertson, 1998). Na decisão final do modelo que representou o ajuste foi realizada pelo coeficiente de correlação obtido pelo método de validação cruzada. Todavia, como segundo critério, adotou-se o maior coeficiente de determinação $\mathrm{r}^{2}$.

Neste trabalho, utilizaram-se também os semivariogramas escalonados com o objetivo de reduzilos à mesma escala, facilitando a comparação entre resultados de diferentes variáveis. Os semivariogramas experimentais foram escalonados por meio da divisão das semivariâncias pela variância amostral de cada variável estudada (Guimarães 1993; Vieira 1997). Com as semivariâncias adimensionalizadas, o efeito pepita expressa diretamente em porcentagem do patamar (semivariância total) o componente aleatório da estrutura de variância. De acordo com Guimarães (1993), verifica-se proporcionalidade quando os semivariogramas escalonados permitem o ajuste de um único modelo para a variável em estudo. 
Os semivariogramas experimentais escalonados foram ajustados ao modelo esférico (Equação 2).

$$
\gamma(h)=\left\{\begin{array}{rc}
C_{0}+C_{1}\left[\frac{3}{2}\left(\frac{h}{a}\right)-\frac{1}{2}\left(\frac{h}{a}\right)^{3}\right], & 0<h \leq a \\
C_{0}+C_{1}, & h \geq a
\end{array}\right.
$$

Em que: $\mathrm{C}_{0}$ é o efeito pepita; $\mathrm{C}_{0}+\mathrm{C}_{1}$, o patamar; $\mathrm{h}$, a distância de separação entre duas observações; a, o alcance de dependência espacial.

\section{RESUltados E DisCUSSÃo}

No estudo, foram realizados cálculos de medidas descritivas (Tabela 1). Ao comparar os valores de média e mediana dos atributos, observando-se que a primeira é sempre maior. Isso ocorre porque a média é mais sensível a valores extremos, de modo geral, os valores de média e mediana do banco de dados foram próximos atendendo às condições de normalidade. Para os valores de assimetria e curtose, a maioria dos atributos apresentaram valores próximos de zero, com exceção para o K, Macro e Micro que apresentaram valores acentuados de curtose. Quanto ao teste de Komogorv-Smirnov, os atributos indicaram normalidade, porém PT, K e P os dados não seguem uma distribuição normal. No entanto, para fins de interpolação por krigagem ordinária, esse não é um pré-requisito (Cressie 1991).

Tabela 1 - Estatística descritiva dos atributos de um Argissolo Vermelho Alítico plíntico sob agrofloresta na região de Humaitá, AM.

\begin{tabular}{lccccccc}
\hline \multicolumn{1}{r}{ Atributos } & Média & Mediana & DP & CV & Assimetria & Curtose & $\mathrm{d}$ \\
\hline${ }^{4} \mathrm{DMG}(\mathrm{mm})$ & 2,09 & 2,03 & 0,71 & 34,89 & $-0,34$ & 0,91 & $*$ \\
$\left.{ }^{5} \mathrm{Ds}(\mathrm{kg} \mathrm{dm})^{-3}\right)$ & 1,31 & 1,31 & 0,094 & 7,20 & 0,10 & 0,49 & $*$ \\
${ }^{6} \mathrm{Macro}(\%)$ & 3,40 & 3,02 & 0,04 & 1,17 & 0,24 & 4,79 & $*$ \\
${ }^{7} \mathrm{Micro}(\%)$ & 30,00 & 31,02 & 0,02 & 11,66 & $-0,06$ & 4,79 & $*$ \\
${ }^{8} \mathrm{PT}(\%)$ & 33,40 & 34,04 & 3,11 & 9,19 & $-0,06$ & $-0,79$ & $\mathrm{~ns}$ \\
$\mathrm{pH}$ & 3,77 & 3,80 & 0,05 & 1,53 & $-0,48$ & 0,80 & $*$ \\
${ }^{9} \mathrm{M} . \mathrm{O}$. & 20,57 & 20,50 & 3,18 & 15,48 & 0,78 & 0,68 & $*$ \\
$\mathrm{Mg}$ & 1,35 & 1 & 0,48 & 35,57 & $-1,69$ & 0,60 & $*$ \\
$\mathrm{~K}$ & 0,09 & 0,11 & 0,09 & 97,94 & 4,30 & 28,08 & $\mathrm{~ns}$ \\
${ }^{10} \mathrm{H}+\mathrm{Al}^{3+}$ & 18,95 & 18,5 & 1,75 & 9,25 & $-0,51$ & $-0,47$ & $*$ \\
${ }^{8} \mathrm{P}$ & 8,18 & 8,00 & 1,55 & 18,96 & 0,57 & 0,39 & $\mathrm{~ns}$ \\
\hline
\end{tabular}

$\mathrm{DP}^{1}=$ desvio padrão; $\mathrm{CV}^{2}=$ coeficiente de variação; $\mathrm{d}^{3}=*$ significativo a $5 \%$ de probabilidade pelo teste KolmogorovSmirnov; ${ }^{4} \mathrm{DMG}=$ diâmetro médio geométrico; ${ }^{5} \mathrm{Ds}=$ densidade do solo; ${ }^{6} \mathrm{Macro}=$ macroporosidade; ${ }^{7}$ Micro $=$ microporosidade; ${ }^{8} \mathrm{PT}=$ porosidade total ${ }^{9} \mathrm{M} . \mathrm{O} .=$ matéria orgânica; $;{ }^{10} \mathrm{H}+\mathrm{Al}^{3+}=$ acidez potencial, ${ }^{11} \mathrm{P}=$ fósforo disponível.

O maior coeficiente de variação (CV) foi encontrado para o K (97,94\%). Esse efeito se deve à grande variação dos teores desse nutriente na área amostrada. De acordo com Warrick e Nielsen (1980), as variáveis DMG, MO, Mg, e P apresentaram CV médio (15,48 a 34,89\%), enquanto a Ds, Macro, Micro, $\mathrm{pH}, \mathrm{H}+\mathrm{Al}^{3+}$ e PT apresentaram CV baixo (1,57 a 9,19\%). Esses valores de CV baixo condizem com uma área mais homogênea para esses atributos. Zanão Júnior et al. (2010), observaram baixo CV para o pH, afirmando ser necessário apenas um pequeno número de subamostras para estimar esse atributo, com boa representatividade. Schlindwein 
e Anghinoni (2000) verificaram CV alto para o K e baixo para pH em água, $\mathrm{H}+\mathrm{Al}$ e matéria orgânica.

Em estudo com variabilidade espacial dos atributos físico-químicos com técnicas de geoestatística, Alves et al. (2014), observou médio CV para a matéria orgânica, baixo CV para o pH. Beckette e Webster (1971) afirmam que $\mathrm{P}, \mathrm{K}$ e Mg são atributos do solo muito alterados pelo manejo, em se tratando de variabilidade.

Com a análise geoestatística realizada por meio de semivariogramas foi constatado que os atributos apresentaram dependência espacial (Tabela 2). A dependência espacial dos atributos estudados, indicada pela relação entre o efeito pepita e o patamar, assim, a variabilidade dos atributos pode ser considerada como não aleatória, uma vez que o espaçamento na grade amostragem foi suficiente para detectar dependência espacial dessas variáveis.

Tabela 2 - Modelos e parâmetros dos atributos de um Argissolo Vermelho Alítico plíntico sob agrofloresta na região de Humaitá, AM.

\begin{tabular}{lccccccc}
\hline Parâmetros & Modelo & $\mathrm{C}_{0}$ & $\mathrm{C}_{0+} \mathrm{C}_{1}$ & $\mathrm{a}(\mathrm{m})$ & $\mathrm{r}^{2}$ & $\mathrm{VC}$ & $\mathrm{C}_{0} / \mathrm{C}_{0+} \mathrm{C}_{1}$ \\
\hline DMG $(\mathrm{mm})$ & Esférico & 0,019 & 0,37 & 17 & 0,79 & 0,83 & 5,09 \\
Ds $\left(\mathrm{kg} \mathrm{dm}^{-3}\right)$ & Esférico & 0,002 & 0,007 & 20 & 0,67 & 0,63 & 28,57 \\
Macro (\%) & Esférico & 1,05 & 2,18 & 33 & 0,75 & 1,17 & 48,16 \\
Micro (\%) & Esférico & 3,13 & 11,59 & 31 & 0,94 & 1,03 & 27,06 \\
$\mathrm{PT}(\%)$ & Esférico & 3,85 & 10,09 & 40 & 0,99 & 0,96 & 35,15 \\
$\mathrm{pH}$ & Esférico & 0,0001 & 0,003 & 15 & 0,75 & 0,72 & 3,33 \\
$\mathrm{M} . \mathrm{O}$. & Esférico & 2,30 & 7,18 & 25 & 0,84 & 0,81 & 32,03 \\
$\mathrm{Mg}$ & Esférico & 0,008 & 0,23 & 29 & 0,70 & 0,60 & 3,47 \\
$\mathrm{~K}$ & Esférico & 0,004 & 0,011 & 61 & 0,99 & 0,55 & 36,36 \\
$\mathrm{H}+\mathrm{Al}$ & Esférico & 1,26 & 3,37 & 38 & 0,78 & 1,22 & 37,38 \\
$\mathrm{P}$ & Esférico & 0,31 & 2,60 & 29 & 0,81 & 0,87 & 11,29 \\
\hline
\end{tabular}

$\mathrm{C}_{0}=$ efeito pepita; ${ }^{2} \mathrm{C}_{1}+\mathrm{C}_{0}=$ variância estrutural; ${ }^{3} \mathrm{a}=$ alcance; ${ }^{4} \mathrm{r}^{2}=$ coeficiente de correlação; ${ }^{5} \mathrm{DE}=$ dependência espacial entre amostras; ${ }^{6} \mathrm{VC}=$ validação cruzada; ${ }^{7} \mathrm{Esf}=$ Esférico;

As validações cruzadas representantes dos modelos ajustados para expressar a dependência espacial dos atributos, evidenciaram um ótimo desempenho, com valores variando de 0,63 a 1,22 (Tabela 2), e coeficientes de determinação $\left(\mathrm{r}^{2}\right)$, variando de 0,67 a 0,99, Azevedo (2004), quando $\left(\mathrm{r}^{2}\right)$ for acima de 50\%, melhor será a estimativa de valores pelo método da krigagem ordinária.

Todos os atributos ajustaram-se ao modelo esférico, trabalhos como Campos et al. (2014) e Oliveira et al. (2015) afirmam que para as propriedades do solo, este modelo é o que promove um melhor ajuste aos semivariogramas experimentais.

Os alcances de dependência espacial foram de 17 a $61 \mathrm{~m}$ para DMG e K, respectivamente, indicando a menor e maior variabilidade. A informação do alcance é importante no planejamento de um experimento, uma vez que quanto maior o alcance maior será a precisão na estimativa por krigagem. Da mesma forma que esse parâmetro indica a linha divisória entre a aplicação da Estatística Clássica ou da Geoestatística (Aquino et al. 2014) e deveria ser considerado em planos de amostragem (Zanão Júnior et al. 2010). Na Figura 2, são apresentados a interação dos atributos em semivariograma escalonado. 
Figura 2 - Parâmetros e modelo do semivariograma escalonado ajustados aos atributos de um Argissolo Vermelho Alítico plíntico sob agrofloresta na região de Humaitá, AM. [modelo (efeito pepita; patamar; GDE; alcance, $\mathbf{R}^{2}$ )].

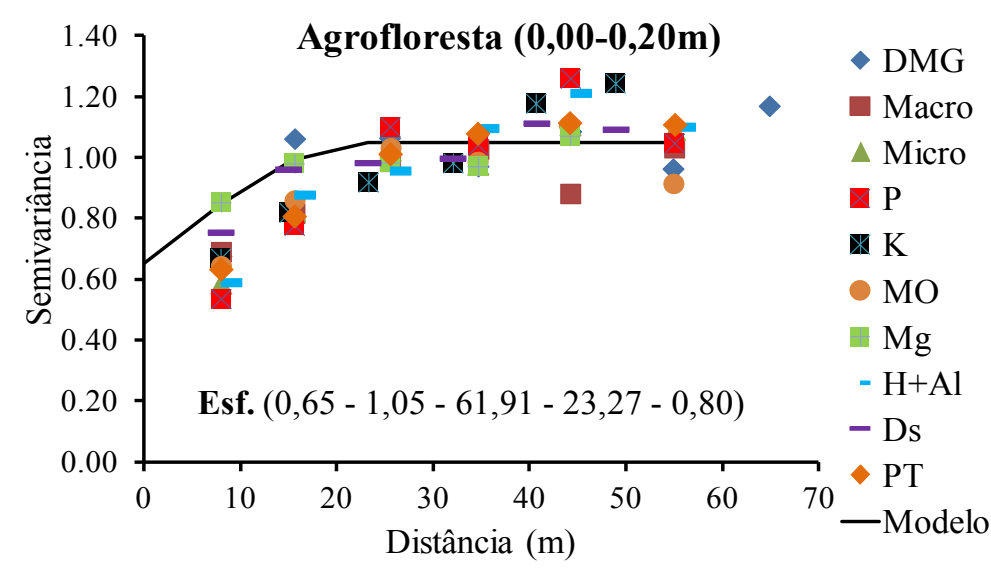

\section{Conclusão}

A densidade do solo apresentou baixos valores de média e coeficiente de variação, enfatizando uma maior homogeneidade dos dados, sendo mais sensíveis à variação do K.

A área em estudo apresentou baixa variação dos atributos, forte a moderada o grau de pendência espacial e a continuidade espacial foi maior que o espaçamento estipulado entre os pontos.

Os sistemas agroflorestas podem ser uma forma metodológica sustentável, pois reconstitui um novo ecossistema com as premissas da floresta primária.

\section{REFERÊNCIAS}

Alvez SMF, Queiroz DM, Alcântara GR e REIS EF. 2014. Variabilidade espacial de atributos físico-químicos do solo usando técnicas de análise de componentes principais. Bioscince Journal, 30 (1): 22-30.

Aquino RL, Campos MCC, Marques Júnior J, Oliveira IA, Mantovanelli BC e Soares MDR. 2014. Geoesatística na avaliação dos atributos físicos em Latossolo son floresta nativa e pastagem na região de Manicoré, Amazonas. Revista Brasileira de Ciência do Solo, 38 (2):397-406.

Aquino RE, Campos MCC, Soares MDR, Oliveria IA, Franciscon U, Silva DMP e Cunha JM. 2016. Chemical soil attributes evaluated by multivariate techniques and geostatistics in the area with agroforestry and sugarcane in Humaitá, AM, Brazil. Bioscience Journal, 32 (1): 61-72.

Azevedo EC. 2004. Uso da geoestatística e de recursos de geoprocessamento no diagnóstico da degradação de um solo argiloso sob pastagem no estado de Mato Grosso. Tese de Doutorado. CampinasUniversidade Estadual de Campinas

Beckett PHT And Webster R. Soil variability: A review. Soils Fert., 34:1.

Bottega EL, Queiroz DM, Pinto FAC, Márcio C e Souza A. 2013.Variabilidade espacial dos atributos do solo 
em sistema de semeadura direta com rotação de culturas no cerrado brasileiro. Revista Ciência Agronômica, 44 (1): $1-9$.

Brasil.1978. Ministério da Agricultura, Pecuária e Abastecimento. Projeto Radam Brasil, folha SB. 20, Purus. Rio de Janeiro, 561p.

Cajazeira JP e Assis Junior RN. de. 2011. Variabilidade espacial das frações primárias e agregados de um Argissolo no Estado do Ceará. Revista Ciência Agronômica, 42 (2) : 258-267.

Cambardella CAE et al. 1994. Field-scale variability of soil properties in Central Iowa. Soil Science Society of American Journal, 58 (5): 1501-1511.

Campos MCC, Soares MDR, Santos LAC, Oliveira IA, Aquino RE e Bergamin AC. 2013. Variabilidade espacial dos atributos físicos em um Argissolo Vermelho sob floresta. Comunicata Scientae, 4 (2): 168-178.

Campos MCC, Soares MDR, Aquino RE, Santos LAC e Mantovanelli BC. 2014. Distribuição espacial da resistência do solo à penetração e teor de água do solo em uma área de agrofloresta na região de Humaitá, AM. Comunicata Scientiae, 5 (4): 509-517.

Castro AP, Fraxe TJ, Santiago JL, Matos RB e Pinto IC. 2009. Os sistemas agroflorestais como alternativa de sustentabilidade em ecossistemas de várzea no Amazonas. Acta Amazônica, 39 (2) :279-288.

Chauvel A, Grimaldi M and TESSIER D. 1991. Changes in soil pore-space distribuition following deforestations and revegetation: An example forma the Central Amazon Basin, Brazil. Forest Ecology and Management, 38 (3): 259-271.

Cunha TJF, Madari BE, Benite VM, Canellas LP, Novotny EH, Moutta RO, Trompowsky PM e Santos GA. 2007. Fracionamento químico da matéria orgânica e características de ácidos húmicos de solos com horizonte a antrópico da amazônia (Terra Preta). Acta Amazonica, 37 (1): p.91-98.

Embrapa. 2011. Empresa Brasileira de Pesquisa Agropecuária. Manual de métodos de análise de solo. Rio de Janeiro. 230p.

Guimarães EC. 1993. Variabilidade espacial da umidade e da densidade do solo em um Latossolo Roxo. Dissertação de Mestrado. Campinas: Universidade Estadual de Campinas.

Isaaks EH e Srivastava RM. 1989. An introduction to applied. Oxford: University Press. 561p.

Kemper WD e Chepil WS.1965. Aggregate stability and size distribution. In: BLACK, C. A. (Ed.) Methods of soil analysis. Madson: ASA, Pt.1, cap. 39, p.499-510.

Marques JDO, Teixeira WG e Reis AM. 2004. Estudo de parâmetros físicos, químicos e hídricos de um Latossolo Amarelo, na região Amazônica. Acta Amazonica, 34 (2): 145-154.

Minitab Release 14.1, Statistical Software. 2000.

Oliveira IA, Campos MCC, Aquino RE, Marques Junior J, Freitas L e Souza ZM. 2014. Semivariograma 
escalonado no planejamento amostral da resistência à penetração e umidade de solo com cana-de-açúcar. Revista Ciências Agrárias, 57 (3): 287-296.

Oliveira IA, Campos MCC, Aquino RE, Silva DMP, Cunha JM, Souza ZM, Soares MDR e Freitas L.2015. Spatial variability of physical attributes of cambisol under cassava cultivation in southern Amazonas. African Journal of Agricultural Research, 10 (49): 4414 -4423.

Robertson, G. P. 1998. GS+ geostatistics for the environmental sciences: GS+ user's guide. Plainwell: Gamma Design Software, 152p.

Schilindwein JA e Anghinoni I. 2000. Variabilidade especial de atributos de fertilidade e amostragem de solo no sistema plantio direto. Revista Brasileira de Ciência do Solo, 24 :85-91.

Silva AS, Lima JSS, Souza GS, Oliveira RB e Silva AF. 2010. Variabilidade espacial do fósforo e das frações granulométricas de um Latossolo Vermelho Amarelo. Revista Ciência Agronômica, 41 (1): 1-8.

Silva DMP, Campos MCC, Alho LC, Cunha JM e Paula Neto P. 2016. Variação espacial da estabilidade de agregados e estoque de carbono em área de terra preta arqueológica sob cultivo de cacau. Revista Brasileira de Agroecologia, 11 (3): 179-187.

Soares MDR, Campos MCC, Souza ZM, Brito WBM, Franciscon U e Castioni GAF. 2015.Variabilidade espacial dos atributos físicos do solo em área de terra preta arqueológica sob pastagem em Manicoré, AM. Revista Ciências Agrárias, 58 (4): 434-441.

Vieira SR. 1997.Variabilidade espacial de argila, silte e atributos químicos em uma parcela experimental de um Latossolo Roxo de Campinas (SP). Bragantia, 56 (1): 181-190.

Vieira TA, Rosa LS, Vasconcelos PCS, Santos MM e Modesto RS. 2007. Sistemas agroflorestais em áreas de agricultores familiares em Igarapé-Açu, Pará: caracterização florística, implantação e manejo. Acta Amazona, 37 (4): 549-558.

Warrick AW, Nielsen DR. 1980. Spatial variability of soil physical properties in the field. In: HILLEL, D. (ed.). Applications of soil physics. New York: Academic, cap. 2, p. 319-344.

Zanão Júnior LA, Lana RMQ, Zanão MPC e Guimarães EC. 2010. Variabilidade espacial de atributos químicos em diferentes profundidades em um Latossolo em sistema de plantio direto. Revista Ceres, 5 (3): 429-438. 